\title{
International Comparisons of the Service Industry- What Can China Learn from Other Countries?
}

\author{
Yueyun(Bill) Chen \\ Department of Business Administration, University of the West, Los Angeles, USA \\ Email: billchen@uwest.edu
}

\begin{abstract}
This paper focuses on analyses of the service industry in different countries over the years. Through this comprehensive study and international comparisons, developing countries like China can learn from others and better develop its service industry. When an economy advances, its service industry will be increasingly important in terms of shares of GDPs and employment. But fast-shrinking of the manufacturing industry and rapid expansion of the service industry may cause serious problems such as being weightless, lowering productivity and suffering from the mid-income trap. To sustain its economic growth, China needs to better expand and develop its service industry, particularly improve and develop social, professional and manufacturing/production services and improve and increase its service export.
\end{abstract}

Keywords: Service industry, economic structure, economic development, China's economy

\section{$1 \quad$ Introduction}

The development of the service industry is crucial to a country's economy. The level of a country's service industry, particularly its ratio of the service industry added-value to the gross domestic product (GDP), indicates the country's economic development stage. In other words, when a country advances from an underdeveloped to developing and then developed economy, its service industry will be increasingly important and its percentage of the GDP and percentage of employment in the service sector will rise.

Many developing economies rely on its manufacturing industry. This is particularly true for exportoriented countries. When a country is underdeveloped or is at the beginning of its development, the total demand for service is low. The priority of the economy is to feed people and provide necessary productsmainly agricultural and manufacturing products. Also, in order to maintain its rapid economic growth, such a country needs to export manufacturing products since it does not have any competitive advantages in its service products. The fact that 70 percent of the world trade is in manufacturing products further confirms that an export-oriented developing economy needs to rely on its manufacturing industry.

After a country has experienced economic development for many years, particularly rapid growth, the country's living standard is raised significantly; then the cost of producing these manufacturing products rises very fast. As a result, their manufacturing products will be less competitive in the world market. When combined with a retrenching world economy, then this kind of manufacturing and export-oriented country will be faced with economic trouble. That is what China has experienced in recent years. In order to stabilize its economy, a country like China must grow its service industry.

China's experience is not unique. Many rapidly growing developing countries such as South Korea and Japan have had similar experiences decades ago. But expanding the service industry and overly-shrinking the manufacturing industry may cause new problems to these developing countries. For example, its economy becomes weightless and less productive and competitive. Therefore, it will be valuable to conduct a comprehensive and dynamic study of the service industry's developments in different countries and provide international comparisons. From this, emerging countries like China can learn from the others' similar experiences.

Each country is different in terms of its available natural resources, population and other factors that will affect and decide its economic structure and international competitive advantages. Both Germany and the 
US are developed countries, but the ratio of the service GDP to its total GDP is about $70 \%$ in Germany and $80 \%$ in the US. Therefore, one can learn from other countries' relevant experiences, but each country needs to develop and implement its economic strategy based on its unique conditions. Particularly an economic strategy needs not only to be based on long-term clear objectives and goals and sound execution, but also to be stable and well balanced. Dramatic changes in economic policies and strategies will cause more troubles than the solutions.

This paper focuses on analyses of the service industry in different countries over the years. The results from this study can help better understand comparative advantages of developed countries like US, Japan, United Kingdom and Germany in some service sectors such as Information and Communications, Finance and Insurance, Profession Service and Public Service. It also shows that developing countries like China lack a necessary balance in the service industry and have several under-developed service sectors. As a result, developed countries have huge overall service trade surplus. Therefore, this study provides directions for developing countries like China on how to expand and develop some service sectors, and improve its overall service industry and its competitiveness. In addition, data collected and outcomes from this study further support the relevant economic theories relating with the service industry as described in the next section.

The rest of this paper is organized as follows: Section 2 reviews literature related to the service industry; Section 3 analyzes relevant data; Section 4 discusses what China can learn from other countries and what it should do; Section 5 is the summary of the paper and Section 6 explains the limitations of this study and possible future research.

\section{Review of Literature on the Service Industry}

An economy consists of three sectors--agriculture, industry and service. The development of the service industry will change a country's economic structure. Such a development is always directly related to a country's economic growth. Therefore, the literature on the service industry development is included in both the economic structure and economic growth. In addition, a country's development of its service industry is related to its industrialization and modernization. Thus there are studies on the service industry from perspectives of the industrialization/ modernization. One can also study the service industry in terms of international trade, particularly globalization.

\subsection{Economic Structure Theory}

Silva and Teixeira (2008) and Kruger (2008) conducted comprehensive surveys of literature on economic structural change. The root of the study on the economic structure can be traced back to Turgot (1766) and Smith (1776). Fisher $(1939,1952)$ defined the economic sectors and indicated that economic structure change occurs when the consumer's preference changes and/or the productivity of the specific sector gains. Kuznet (1966) gave arguments on why a country's economic structure changes and emphasized the four factors that will lead to the structural change--increasing demand for non-agricultural products, higher incomes and demand for importing products, international trade with less developed countries, and technological progress.

Baumol (1967) raised a serious issue on economic structural change called 'cost disease.' It involves a rise of salaries in jobs that have not experienced any labor productivity increase. Although the productivity in the service is not increased, firms in this industry need to pay more in order to retain and attract workers because the productivity in other sectors (industry sector) has risen and therefore salaries rise there. The associated outcome from this cost disease is that productivity growth in the whole economy will slow down when resources shift to service industry. Oulton (2001) showed that the above outcome is true only for service industries which provide final goods but not true for the ones providing intermediate goods.

China's economic development relies on its economic structure change and this change, in return, builds up the foundation for its economic growth because more labors have been moved from the agriculture sector to the industrial and service sectors (Chen 2015; Zhu 2012). In 1990, the agriculture industry generated 
about $27.11 \%$ of its total GDP, the industrial industry was $41.34 \%$ and service $32.54 \%$; in 2000 , these were $15.06 \%, 45.92 \%$ and $39.02 \%$, respectively; and in 2010, they were $10.10 \%, 46.67 \%$ and $43.24 \%$ (Chen 2015). Last year, they were $9.0 \%, 40.5 \%$ and $50.5 \%$, respectively. The agriculture industry used to employ the most people and now the service sectors have the most employees. So China's experience shows that the service industry grows when a country's economy advances.

\subsection{Economic Growth Theory}

What is the relationship between economic growth and structure change? According to neoclassical growth theory (Solow 1956, 1957) and new growth theory (Lucas 1988; Romer 1986, 1990), technological progress is the only main factor contributing to economic growth. Economic growth warrants transfer of resources from low-productivity primary production to high-productivity secondary sectors. At the mature stage, resources then are transferred from secondary to tertiary sectors (Clark 1958, Rostow 1960, Lewis 1972). That is what the world economy has generally experienced, i.e. when the economy advances, more resources will be shifted from the agricultural sector to the industry sector, and then to the service sector.

Pelka (2005) developed theoretical models and found that only the economic growth process promotes structural change. However, Dietrick (2009) used OECD data from 1960-2004 and found evidence that economic structure change causes aggregate economic growth, and conversely aggregate growth leads to economic structure change. In other words, the relationship between economic growth and structure change is dynamic and interactive.

China as a developing country experienced rapid economic growth for more than two decades and particularly after its entry into the WTO in 2001. China's miracle benefited from its active participations in the globalization and more specifically from its increasing exports and governments investments (Lin, et al. 2003, Lin 2012, Rasiah, et al. 2013). The fast service industry development in China shows that the economic growth leads to the shifts of resources among three industries.

\subsection{Industrialization/Modernization and Service Industry}

Industrialization accelerates technological progress and improves productivity. That further leads to rapid shifts in production and labor from primary/agriculture to the secondary/ industry sector. Consequently, the service sector rises which support the arguments stated by Kuznet (1966). Hansen and Prescott (2002) showed the importance of technology to growth after industrial revolution. Sekhon, et al. (2016) showed that resources commitment to the service industry raises its productivity through employees' perceptions of the job safety.

Modern economic growth depends on the shift from a pre-industrial land-intensive Malthusian technology with decreasing return to labor, to a Solowian constant returns-to-scale technology, with both capital and labor as inputs. Post-industrial theory (Touraine 1969, Bell 1973, Castells and Aoyama 1994) states that the more advanced an economy, the more its employment and production would be focused on services. The one main source of such shifts is the information and its technology. Knowledge and information are the main sources of productivity and growth, and so the modern economy has become the information economy (Porat 1977, Nelson 1981, Monk 1989, Denison 1985, Sautter 1976, Baumol et al. 1989).

Castells and Aoyama (1994) conducted an empirical study of employment structure in G-7 countries from 1920-1990 and concluded that the post-industrialization led to:

"the phasing out of agricultural employment, the steady decline of manufacturing employment, the rise of both producer services and social services, the increasing diversification of service activities as sources of jobs, the rapid rise of managerial, professional and technical jobs, the formation of a white-collar proletariat of semi-skilled clerical and sales workers, a substantial and relatively stable share of employment in the retail trade, and the overall upgrading of the occupational structure over time, with an increasing share going to occupations that require higher skills and advanced education."

The authors further proposed two different informational models: (1) a "service economy model" represented by the US, UK and Canada. These countries have had rapid decline of manufacturing 
employment after 1970s and (2) an "info-industrial model" represented by Japan and Germany. In these two countries, the share of manufacturing employment has reduced significantly, but it has maintained at a relatively high level (about 25\%). Other G-7 countries fill in between these two models.

Although the service industry becomes increasingly important in a developed economy, many services and its jobs are directly related with the manufacturing industry. Cohen and Zysman (1987) argued that the post-industrial US economy is not the service economy but is a "myth". They estimated that $24 \%$ of the US GDP comes from the value added by manufacturing firms and another $25 \%$ of GDP comes from the contribution of services directly linked to manufacturing.

There are different types of services, including finance, real estate, health, and education. Stiglitz (2012) emphasized that the US needs to develop a creative economy and transfer jobs from manufacturing to services that people want - into productive activities that increase living standards, not those that increase risk and inequality. He particularly suggested that governments should invest in education and support basic research. Potts and Mandeville (2007) emphasized the importance of innovation in services, and they argued that services firms should better use information and communication technologies to coordinate service production and delivery.

China has experienced and benefited from increasing industrialization. Industrialization enables China to use modern technologies and improve its productivity. The service industry directly benefits from the industrialization because it expands or adds new service sectors like Financial Service, and Information and Communications as well as raising the service productivity.

\subsection{International Trade and Service Industry}

As Kuznet (1966) argued, increasing income will raise demand for importing products, and also trade with less developed countries will affect a country's economic structure. As we have witnessed, developed countries produce less and less manufacturing products and they buy these products from developing or underdeveloped countries. The service industry becomes increasingly significant and important in developed countries along with rapid increase in international trade and globalization (Wirtz, et al. 2015).

International trade in service has been increasing in the past decades. Some services must be localized such as haircuts and facility maintenance. But others can be traded. Financial services now has been highly internationalized and globalized. Western banks and insurers are successfully expanding their businesses in developing countries. Productivity and competitiveness are the keys to the service trade.

Globalization has made the world more flat. Besides the significant increase of the world commodity trade, globalization brings more foreign direct investments and makes financial services more important.

China's fast economic growth in the past two decades largely benefited from increasing globalization and particularly its exports. In 2006, China's export-to-GDP ratio was 36\% (Chen 2015). Its service industry also benefited from globalization and international trade. China attracted most FDIs in the past many years and now China is increasingly investing in other countries and moving toward FDI outwards.

\section{Data Analyses and Implications}

Unless otherwise mentioned, all data sources used in this paper are from the World Bank at www.worldbank.org. Besides China, Russia, India and Brazil are selected because they are BRIC countries; besides US, United Kingdom, Japan, and Germany, South Korean and Singapore are also included because they are Asian countries and experienced fast economic growth during 1970 and 80s.

As explained in the above section, the development of the service industry is related with the whole economy and especially the economic structure. It is also related with the economic growth and international trade. The distribution of the service sectors is particularly important in order to understand a country's strength and competitiveness in the service industry. Hence, relevant data are collected and compared in the following Sections 3.1-3.6 for these selected countries as well as the whole world. In addition, in Section 3.7, correlations among selected variables are calculated and explained. 


\subsection{The World Total GDP and Service GDP}

Table 1. World total GDP and service GDP

\begin{tabular}{llllll}
\hline & $\begin{array}{l}\text { World Total GDP } \\
\text { (Current US } \$ \text { ) }\end{array}$ & $\begin{array}{l}\text { of } \\
\text { Industry } \\
\text { GDP }\end{array}$ & $\begin{array}{l}\text { Total World } \\
\text { Industry GDP }\end{array}$ & $\begin{array}{l}\text { \% of } \\
\text { Service } \\
\text { GDP }\end{array}$ & $\begin{array}{l}\text { World Total } \\
\text { Service GDP }\end{array}$ \\
\hline 1995 & $30,332,592,675,000$ & 32.8 & $9,949,090,397,400$ & 60.8 & $18,442,216,346,400$ \\
2000 & $32,981,132,788,000$ & 29.3 & $9,663,471,906,884$ & 66.7 & $21,998,415,569,596$ \\
2005 & $46,468,942,222,000$ & 28.3 & $13,150,710,648,826$ & 68.3 & $31,738,287,537,626$ \\
2006 & $50,334,896,708,000$ & 28.6 & $14,395,780,458,488$ & 68.2 & $34,328,399,554,856$ \\
2007 & $56,696,185,105,000$ & 28.4 & $16,101,716,569,820$ & 68.4 & $38,780,190,611,820$ \\
2008 & $62,170,569,711,000$ & 28.1 & $17,469,930,088,791$ & 68.9 & $42,835,522,530,879$ \\
2009 & $58,885,873,283,000$ & 26.4 & $15,545,870,546,712$ & 70.6 & $41,573,426,537,798$ \\
2010 & $64,552,742,595,000$ & 27 & $17,429,240,500,650$ & 70 & $45,186,919,816,500$ \\
2011 & $71,448,828,400,000$ & 27.2 & $19,434,081,324,800$ & 69.7 & $49,799,833,394,800$ \\
2012 & $72,908,414,851,000$ & 26.9 & $19,612,363,594,919$ & 70.1 & $51,108,798,810,551$ \\
\hline
\end{tabular}

The above table shows the world total GDP, world total service GDP, and percentage of the service GDP to its total GDP since 1995.

The world total service GDP was about $70 \%$ of the world's total GDP in 2012; it was about $67 \%$ in 2000 and about $60 \%$ in 1995 . Overall, the service industry has been increasingly important.

\subsection{The World Total Trade and Total Service Trade}

Table 2. World total trade and service trade

\begin{tabular}{|c|c|c|c|c|c|c|c|c|c|}
\hline & $\begin{array}{l}\text { World Total } \\
\text { GDP } \\
\text { (Current } \\
\text { US1,000\$) }\end{array}$ & $\begin{array}{l}\% \text { of } \\
\text { Total } \\
\text { Trade to } \\
\text { GDP }\end{array}$ & $\begin{array}{l}\text { World Total } \\
\text { Trade }\end{array}$ & $\begin{array}{l}\% \text { of } \\
\text { Service } \\
\text { Trade to } \\
\text { Total } \\
\text { GDP } \\
\end{array}$ & $\begin{array}{l}\text { World } \\
\text { Total } \\
\text { Service } \\
\text { Trade }\end{array}$ & $\begin{array}{l}\% \text { of } \\
\text { Service } \\
\text { Trade to } \\
\text { Total } \\
\text { Trade } \\
\end{array}$ & $\begin{array}{l}\text { Total World } \\
\text { Export } \\
\text { (Current } \\
\text { US1,000\$) }\end{array}$ & $\begin{array}{l}\text { Total World } \\
\text { Service Export } \\
\text { (Current \$US) }\end{array}$ & $\begin{array}{l}\% \text { of } \\
\text { Service } \\
\text { Export to } \\
\text { Total } \\
\text { Export } \\
\end{array}$ \\
\hline 2005 & $46,468,942,222$ & 55.12 & $25,613,680,953$ & 10.87 & $5,051,174,020$ & 19.72 & $12,781,517,318$ & $2,583,043,928,860$ & 20.21 \\
\hline 2006 & $50,334,896,708$ & 57.92 & $29,153,972,173$ & 11.29 & $5,682,809,838$ & 19.49 & $14,706,110,101$ & $2,922,293,893,454$ & 19.87 \\
\hline 2007 & $56,696,185,105$ & 58.70 & $33,280,660,657$ & 11.92 & $6,758,185,265$ & 20.31 & $17,149,064,383$ & $3,486,259,004,445$ & 20.33 \\
\hline 2008 & $62,170,569,711$ & 60.83 & $37,818,357,555$ & 12.29 & $7,640,763,017$ & 20.20 & $19,650,654,712$ & $3,914,341,409,167$ & 19.92 \\
\hline 2009 & $58,885,873,283$ & 52.38 & $30,844,420,426$ & 11.69 & $6,883,758,587$ & 22.32 & $15,755,009,644$ & $3,523,647,209,987$ & 22.37 \\
\hline 2010 & $64,552,742,595$ & 57.42 & $37,066,184,798$ & 11.66 & $7,526,849,787$ & 20.31 & $18,773,432,137$ & $3,852,938,794,679$ & 20.52 \\
\hline 2011 & $71,448,828,400$ & 61.11 & $43,662,379,035$ & 11.80 & $8,430,961,751$ & 19.31 & $22,180,434,646$ & $4,328,262,400,301$ & 19.51 \\
\hline 2012 & $72,908,414,851$ & 60.66 & $44,226,244,449$ & 11.80 & $8,603,192,952$ & 19.45 & $22,433,853,716$ & $4,470,515,252,879$ & 19.93 \\
\hline
\end{tabular}

Table 2 provides the world total trade, world total service trade, and service export as well as the relevant percentage of the service trade and service export.

The world total trade has been increasing over the years. It was about $61 \%$ of the world's total GDP in 2012 while it was about $55 \%$ in 2005. According to the World Bank data, the world's total service trade was about $20 \%$ of the total trade, and the service export was also about $20 \%$ of the world's total export in 2012. However, based on the analysis by McKinsey (2012), the world's service export composes about $30 \%$ of the world's total export. 


\subsection{GDP per Capita in China and Other Countries}

Table 3. GDP and GDP per capita in China and other countries (data from IMF)

\begin{tabular}{|c|c|c|c|c|c|c|c|c|c|c|}
\hline & \multicolumn{2}{|c|}{ China } & \multicolumn{2}{|c|}{ US } & \multicolumn{2}{|c|}{ Germany } & \multicolumn{2}{|c|}{ Japan } & \multicolumn{2}{|c|}{ United Kingdom } \\
\hline & $\begin{array}{c}\text { GDP- } \\
\text { Bil US\$ } \\
\end{array}$ & $\begin{array}{c}\text { Per } \\
\text { Capita } \$ \\
\end{array}$ & $\begin{array}{c}\text { GDP-Bil } \\
\text { US\$ }\end{array}$ & $\begin{array}{c}\text { Per } \\
\text { Capita } \$ \\
\end{array}$ & $\begin{array}{c}\text { GDP- } \\
\text { Bil US\$ }\end{array}$ & $\begin{array}{c}\text { Per } \\
\text { Capita } \$ \\
\end{array}$ & $\begin{array}{c}\text { GDP- } \\
\text { Bil US\$ }\end{array}$ & $\begin{array}{c}\text { Per } \\
\text { Capita } \$ \\
\end{array}$ & $\begin{array}{c}\text { GDP-Bil } \\
\text { US\$ }\end{array}$ & $\begin{array}{c}\text { Per } \\
\text { Capita } \$ \\
\end{array}$ \\
\hline 1980 & 303.37 & 307.35 & 2862.48 & 12575.57 & 826.14 & 10699.04 & 1086.99 & 9311.79 & 542.45 & 9629.89 \\
\hline 1985 & 307.02 & 290.05 & 4346.75 & 18231.83 & 639.70 & 8358.02 & 1384.53 & 11464.24 & 468.96 & 8292.22 \\
\hline 1990 & 390.28 & 341.35 & 5979.55 & 23913.66 & 1547.03 & 19500.58 & 3103.70 & 25139.58 & 1024.56 & 17900.27 \\
\hline 1995 & 727.95 & 601.01 & 7664.05 & 28762.68 & 2525.02 & 30861.58 & 5333.93 & 42516.46 & 1181.01 & 20353.45 \\
\hline 2000 & 1198.48 & 945.60 & 10289.73 & 36450.14 & 1891.93 & 22999.57 & 4731.20 & 37303.81 & 1496.61 & 25415.32 \\
\hline 2005 & 2256.92 & 1726.05 & 13095.43 & 44224.13 & 2771.06 & 33613.84 & 4571.87 & 35780.57 & 2324.18 & 38585.28 \\
\hline 2006 & 2712.92 & 2063.87 & 13857.90 & 46358.36 & 2905.45 & 35296.70 & 4356.75 & 34076.75 & 2486.60 & 41043.81 \\
\hline 2007 & 3494.24 & 2644.56 & 14480.35 & 47963.56 & 3328.59 & 40484.99 & 4356.35 & 34038.35 & 2858.18 & 46866.10 \\
\hline 2008 & 4519.95 & 3403.53 & 14720.25 & 48307.78 & 3640.73 & 44397.84 & 4849.19 & 37865.07 & 2709.57 & 44131.29 \\
\hline 2009 & 4990.53 & 3739.62 & 14417.95 & 46906.90 & 3306.78 & 40424.06 & 5035.14 & 39321.22 & 2217.43 & 35885.34 \\
\hline 2010 & 5930.39 & 4422.66 & 14958.30 & 48294.15 & 3310.60 & 40495.85 & 5495.39 & 42916.74 & 2296.93 & 36891.36 \\
\hline 2011 & 7321.99 & 5434.36 & 15533.83 & 49797.25 & 3631.44 & 45207.64 & 5905.63 & 46175.36 & 2464.64 & 38945.08 \\
\hline 2012 & 8229.38 & 6077.65 & 16244.58 & 51708.98 & 3427.85 & 42569.47 & 5937.77 & 46530.38 & 2484.45 & 38999.21 \\
\hline $\begin{array}{l}\mathbf{2 0 1 3} \\
(1980- \\
2013)\end{array}$ & 9181.38 & 6747.23 & 16799.70 & 53101.01 & 3635.96 & 44999.50 & 4901.53 & 38491.35 & 2535.76 & 39567.41 \\
\hline change & 30.27 & 21.95 & 5.87 & 4.22 & 4.40 & 4.21 & 4.51 & 4.13 & 4.67 & 4.11 \\
\hline
\end{tabular}

Table 3. Continued

\begin{tabular}{|c|c|c|c|c|c|c|c|c|c|c|}
\hline & \multicolumn{2}{|c|}{ South Korea } & \multicolumn{2}{|c|}{ India } & \multicolumn{2}{|c|}{ Singapore } & \multicolumn{2}{|c|}{ Russia } & \multicolumn{2}{|c|}{ Brazil } \\
\hline & $\begin{array}{c}\text { GDP-Bil } \\
\text { US\$ }\end{array}$ & $\begin{array}{c}\text { Per } \\
\text { Capita } \$ \\
\end{array}$ & $\begin{array}{c}\text { GDP-Bil } \\
\text { US\$ }\end{array}$ & $\begin{array}{c}\text { Per } \\
\text { Capita } \$ \\
\end{array}$ & $\begin{array}{c}\text { GDP-Bil } \\
\text { US\$ }\end{array}$ & $\begin{array}{c}\text { Per } \\
\text { Capita } \$ \\
\end{array}$ & $\begin{array}{c}\text { GDP-Bil } \\
\text { US\$ }\end{array}$ & $\begin{array}{c}\text { Per } \\
\text { Capita } \$ \\
\end{array}$ & $\begin{array}{c}\text { GDP-Bil } \\
\text { US } \$\end{array}$ & $\begin{array}{c}\text { Per } \\
\text { Capita } \$ \\
\end{array}$ \\
\hline 1980 & 64.39 & 1688.84 & 181.42 & 265.91 & 12.05 & 4990.19 & & & 148.92 & 1256.01 \\
\hline 1985 & 98.50 & 2413.94 & 237.62 & 313.07 & 18.46 & 6748.29 & & & 231.76 & 1742.55 \\
\hline 1990 & 270.41 & 6307.66 & 326.61 & 385.41 & 38.84 & 12745.06 & & & 465.01 & 3172.10 \\
\hline 1995 & 531.14 & 11778.76 & 366.60 & 391.25 & 87.06 & 24702.00 & 313.45 & 2113.63 & 769.74 & 4844.95 \\
\hline 2000 & 533.39 & 11346.66 & 476.64 & 463.12 & 94.31 & 23413.77 & 259.70 & 1775.13 & 644.73 & 3764.21 \\
\hline 2005 & 844.87 & 17550.88 & 834.22 & 748.85 & 125.43 & 29403.39 & 763.70 & 5310.88 & 882.04 & 4809.85 \\
\hline 2006 & 951.77 & 19676.11 & 949.12 & 839.93 & 146.01 & 33174.49 & 989.93 & 6912.93 & 1089.26 & 5869.97 \\
\hline 2007 & 1049.24 & 21590.17 & 1238.48 & 1080.70 & 178.26 & 38848.26 & 1299.70 & 9101.56 & 1366.85 & 7284.37 \\
\hline 2008 & 931.41 & 19028.07 & 1223.21 & 1052.67 & 190.32 & 39326.78 & 1660.85 & 11630.58 & 1653.54 & 8720.60 \\
\hline 2009 & 834.06 & 16958.65 & 1365.34 & 1158.91 & 190.16 & 38127.43 & 1222.65 & 8567.94 & 1622.31 & 8472.44 \\
\hline 2010 & 1014.89 & 20540.18 & 1708.54 & 1430.19 & 233.29 & 45953.53 & 1524.92 & 10671.21 & 2142.91 & 11088.60 \\
\hline 2011 & 1114.47 & 22388.40 & 1880.10 & 1552.55 & 272.32 & 52533.15 & 1893.79 & 13252.56 & 2474.64 & 12693.89 \\
\hline 2012 & 1129.60 & 22590.16 & 1858.75 & 1514.63 & 284.30 & 53516.04 & 2004.25 & 14015.75 & 2247.75 & 11437.39 \\
\hline $\begin{array}{c}2013 \\
(1980- \\
2013)\end{array}$ & 1221.80 & 24328.98 & 1870.65 & 1504.54 & 295.74 & 54775.53 & 2118.01 & 14818.64 & 2242.85 & 11310.88 \\
\hline change & 18.98 & 14.41 & 10.31 & 5.66 & 24.55 & 10.98 & 6.76 & 7.01 & 15.06 & 9.01 \\
\hline
\end{tabular}


A country's economic development level will affect its economic structure, particularly its service sector's GDP and employment shares. Table 3 shows the total GDP and GDP per capita of selected countries from 1980-2013.

During 1980-2013, China's GDP was increased by 30.27 times and its GDP per capita by 21.95 times; US total GDP increased by 5.87 times and per capita by 4.22; Germany total GDP by 4.40 times and per capita by 4.21; Japan's increases were 4.51 and 4.13; UK was 4.67 and 4.11; Korea was 18.98 and 14.41, and Singapore was 24.55 and 10.98. At the same time, other BRIC countries experienced the following rates: India's GDP increased by 10.31 and per capita by 5.66; Russian increased by 6.76 and 7.01 (from 1995 to 2012) and Brazil increased by 15.06 and 9.01, respectively.

Based on the above statistics, China had the highest percentage increases in total GDP and GDP per capita during these years. UK had the lowest GDP per capita increase and Germany had the lowest GDP increase during the time period.

Also, among all of these countries, in 2013 Singapore and the US had the highest GDP per capita (above $\$ 50,000)$; Germany, UK and Japan were the next group, each with about $\$ 40,000$ GDP per capita; Russia and Brazil were above $\$ 10,000$ GDP per capita; China was about $\$ 7,000$ GDP per capita; and India had the lowest with about $\$ 1,500$ per capita.

\subsection{China and Other Countries' Service GDP and Service Employment}

Table 4 shows the percentage of service GDP to its total GDP and percentage of service employment to its total employment in China, US, UK, Japan, South Korea, Singapore, India, Russia, and Brazil.

Table 4. GDP and Employment in Service Industry

\begin{tabular}{|c|c|c|c|c|c|c|c|c|c|c|c|}
\hline & & \multicolumn{2}{|c|}{ China } & \multicolumn{2}{|l|}{ US } & \multicolumn{2}{|c|}{ Germany } & \multicolumn{2}{|c|}{ Japan } & \multicolumn{2}{|c|}{ United Kingdom } \\
\hline & & $\begin{array}{l}\% \\
\text { GDP }\end{array}$ & $\begin{array}{l}\% \\
\text { Jobs }\end{array}$ & $\begin{array}{l}\% \\
\text { GDP }\end{array}$ & $\begin{array}{l}\% \\
\text { Jobs }\end{array}$ & $\begin{array}{l}\% \\
\text { GDP }\end{array}$ & $\begin{array}{l}\% \\
\text { Jobs }\end{array}$ & $\begin{array}{l}\% \\
\text { GDP }\end{array}$ & $\begin{array}{l}\% \\
\text { Jobs }\end{array}$ & $\% \mathrm{GDP}$ & $\%$ Jobs \\
\hline \multirow[t]{3}{*}{1980} & Agriculture & 30.17 & 68.69 & 2.90 & 3.59 & 2.40 & 5.20 & 3.08 & 10.39 & 2.10 & 2.59 \\
\hline & Industry & 48.22 & 18.20 & 33.50 & 30.79 & 41.10 & 42.90 & 39.06 & 35.29 & 40.70 & 37.20 \\
\hline & Service & 21.60 & 13.10 & 63.60 & 65.69 & 56.50 & 51.90 & 57.86 & 54.00 & 57.20 & 58.90 \\
\hline \multirow[t]{3}{*}{1985} & Agriculture & 28.44 & 62.40 & 2.40 & 3.09 & 1.90 & 4.50 & 2.69 & 8.80 & 1.70 & 2.50 \\
\hline & Industry & 42.88 & 20.79 & 30.90 & 28.29 & 39.00 & 40.10 & 38.22 & 34.90 & 38.30 & 31.20 \\
\hline & Service & 28.67 & 16.79 & 66.70 & 68.59 & 59.00 & 55.40 & 59.07 & 56.00 & 60.00 & 64.90 \\
\hline \multirow[t]{3}{*}{1990} & Agriculture & 27.11 & 60.09 & 2.10 & 2.90 & 1.50 & 3.50 & 2.12 & 7.19 & 1.44 & 2.09 \\
\hline & Industry & 41.34 & 21.39 & 27.90 & 26.39 & 37.30 & 39.00 & 38.05 & 34.09 & 31.41 & 32.29 \\
\hline & Service & 31.54 & 18.50 & 70.10 & 70.69 & 61.20 & 57.60 & 59.82 & 58.20 & 67.14 & 64.90 \\
\hline \multirow[t]{3}{*}{1995} & Agriculture & 19.96 & 52.20 & 1.61 & 2.90 & 1.09 & 3.20 & 1.73 & 5.70 & 1.57 & 2.00 \\
\hline & Industry & 47.18 & 23.00 & 26.31 & 24.30 & 32.34 & 36.00 & 32.71 & 33.60 & 30.28 & 27.30 \\
\hline & Service & 32.86 & 24.80 & 72.08 & 72.90 & 66.56 & 60.80 & 65.56 & 60.40 & 68.15 & 70.20 \\
\hline \multirow[t]{3}{*}{2000} & Agriculture & 15.06 & 50.00 & 1.19 & 2.60 & 1.11 & 2.60 & 1.59 & 5.10 & 0.90 & 1.50 \\
\hline & Industry & 45.92 & 22.50 & 23.44 & 23.20 & 30.51 & 33.50 & 31.00 & 31.20 & 26.80 & 25.10 \\
\hline & Service & 39.02 & 27.50 & 75.37 & 74.30 & 68.38 & 63.80 & 67.42 & 63.10 & 72.30 & 73.10 \\
\hline \multirow[t]{3}{*}{2005} & Agriculture & 12.12 & 44.80 & 1.21 & 1.60 & 0.80 & 2.40 & 1.22 & 4.40 & 0.63 & 1.30 \\
\hline & Industry & 47.37 & 23.80 & 22.19 & 20.60 & 29.30 & 29.80 & 28.05 & 27.90 & 23.62 & 22.20 \\
\hline & Service & 40.51 & 31.40 & 76.60 & 77.80 & 69.90 & 67.80 & 70.73 & 66.40 & 75.75 & 76.30 \\
\hline \multirow[t]{3}{*}{2006} & Agriculture & 11.11 & 42.60 & 1.04 & 1.50 & 0.82 & 2.30 & 1.18 & 4.30 & 0.59 & 1.30 \\
\hline & Industry & 47.95 & 25.20 & 22.24 & 20.80 & 30.14 & 29.70 & 28.06 & 28.00 & 23.62 & 22.00 \\
\hline & Service & 40.94 & 32.20 & 76.72 & 77.70 & 69.04 & 68.10 & 70.76 & 66.60 & 75.78 & 76.40 \\
\hline \multirow[t]{2}{*}{2007} & Agriculture & 10.77 & 40.80 & 1.13 & 1.40 & 0.87 & 2.20 & 1.15 & 3.90 & 0.63 & 1.40 \\
\hline & Industry & 47.34 & 26.80 & 21.99 & 20.60 & 30.50 & 29.90 & 28.15 & 27.40 & 23.01 & 22.30 \\
\hline
\end{tabular}




\begin{tabular}{llllllllllll}
\hline \multirow{2}{*}{2008} & Service & 41.89 & 32.40 & 76.88 & 78.00 & 68.63 & 67.80 & 70.70 & 67.20 & 76.36 & 76.00 \\
& Agriculture & 10.73 & 39.60 & 1.22 & 1.50 & 0.96 & 1.80 & 1.14 & 3.80 & 0.69 & 1.10 \\
& Industry & 47.45 & 27.20 & 21.13 & 19.90 & 30.13 & 29.30 & 27.43 & 26.90 & 22.63 & 21.90 \\
& Service & 41.82 & 33.20 & 77.65 & 78.60 & 68.91 & 68.90 & 71.43 & 67.80 & 76.68 & 76.60 \\
\multirow{2}{2009}{} & Agriculture & 10.33 & 38.10 & 1.12 & 1.50 & 0.75 & 1.70 & 1.16 & 3.90 & 0.58 & 1.10 \\
& Industry & 46.24 & 27.80 & 19.58 & 17.10 & 27.82 & 28.80 & 25.94 & 25.90 & 21.26 & 19.50 \\
& Service & 43.43 & 34.10 & 79.30 & 80.90 & 71.43 & 69.50 & 72.90 & 69.00 & 78.17 & 78.70 \\
$\mathbf{2 0 1 0}$ & Agriculture & 10.10 & 36.70 & 1.19 & 1.60 & 0.80 & 1.60 & 1.18 & 3.70 & 0.68 & 1.20 \\
& Industry & 46.67 & 28.70 & 19.79 & 16.70 & 30.23 & 28.40 & 27.46 & 25.30 & 21.52 & 19.10 \\
& Service & 43.24 & 34.60 & 79.02 & 81.20 & 68.97 & 70.00 & 71.36 & 69.70 & 77.80 & 78.90 \\
\hline
\end{tabular}

Table 4. Continued

\begin{tabular}{|c|c|c|c|c|c|c|c|c|c|c|c|}
\hline & & \multicolumn{2}{|c|}{ South Korea } & \multicolumn{2}{|l|}{ India } & \multicolumn{2}{|c|}{ Singapore } & \multicolumn{2}{|c|}{ Russia } & \multicolumn{2}{|c|}{ Brazil } \\
\hline & & $\begin{array}{l}\% \\
\text { GDP }\end{array}$ & $\%$ Jobs & $\begin{array}{l}\% \\
\text { GDP }\end{array}$ & $\begin{array}{l}\% \\
\text { Jobs }\end{array}$ & $\begin{array}{l}\% \\
\text { GDP }\end{array}$ & $\begin{array}{l}\% \\
\text { Jobs }\end{array}$ & $\begin{array}{l}\% \\
\text { GDP }\end{array}$ & $\begin{array}{l}\% \\
\text { Jobs }\end{array}$ & $\begin{array}{l}\% \\
\text { GDP }\end{array}$ & $\begin{array}{l}\% \\
\text { Jobs }\end{array}$ \\
\hline \multirow[t]{3}{*}{1980} & Agriculture & 15.11 & 34.00 & 35.38 & & 1.56 & 1.29 & & & 11.01 & \\
\hline & Industry & 34.16 & 29.00 & 24.28 & & 36.22 & 35.70 & & & 43.82 & \\
\hline & Service & 50.72 & 37.00 & 40.32 & & 62.20 & 62.59 & & & 45.16 & \\
\hline \multirow[t]{3}{*}{1985} & Agriculture & 12.51 & 24.89 & 30.89 & & 0.95 & 0.69 & & & 11.54 & 28.60 \\
\hline & Industry & 36.10 & 30.79 & 25.69 & & 33.44 & 35.20 & & & 45.31 & 22.10 \\
\hline & Service & 51.38 & 44.29 & 43.40 & & 65.59 & 64.09 & & & 43.14 & 49.29 \\
\hline \multirow[t]{3}{*}{1990} & Agriculture & 8.21 & 17.89 & 29.02 & & 0.33 & & 16.81 & 13.89 & 8.10 & 22.79 \\
\hline & Industry & 38.18 & 35.40 & 26.49 & & 32.34 & & 48.35 & 40.00 & 38.69 & 22.70 \\
\hline & Service & 53.59 & 46.70 & 44.48 & & 67.32 & & 35.03 & 45.59 & 53.21 & 54.50 \\
\hline \multirow[t]{3}{*}{1995} & Agriculture & 5.82 & 12.40 & 26.26 & & 0.16 & 0.20 & 7.16 & 15.70 & 9.85 & 26.10 \\
\hline & Industry & 38.38 & 33.30 & 27.40 & & 33.75 & 31.00 & 36.96 & 34.00 & 40.00 & 19.60 \\
\hline & Service & 55.80 & 54.30 & 46.34 & & 66.09 & 68.80 & 55.88 & 50.00 & 50.15 & 54.30 \\
\hline \multirow[t]{3}{*}{2000} & Agriculture & 4.39 & 10.60 & 23.02 & 59.90 & 0.10 & & 6.43 & 14.50 & 5.60 & \\
\hline & Industry & 38.09 & 28.10 & 26.00 & 16.00 & 34.83 & & 37.95 & 28.40 & 27.73 & \\
\hline & Service & 57.51 & 61.20 & 50.98 & 24.00 & 65.07 & & 55.62 & 57.10 & 66.67 & \\
\hline \multirow[t]{3}{*}{2005} & Agriculture & 3.15 & 7.90 & 18.81 & 55.80 & 0.06 & 1.10 & 4.97 & 10.20 & 5.71 & 20.50 \\
\hline & Industry & 37.50 & 26.80 & 28.13 & 19.00 & 32.36 & 21.70 & 38.08 & 29.80 & 29.27 & 21.40 \\
\hline & Service & 59.36 & 65.20 & 53.06 & 25.20 & 67.58 & 77.30 & 56.96 & 60.00 & 65.02 & 57.90 \\
\hline \multirow[t]{3}{*}{2006} & Agriculture & 2.99 & 7.70 & 18.29 & & 0.05 & 1.30 & 4.52 & 10.00 & 5.48 & 19.30 \\
\hline & Industry & 36.86 & 26.30 & 28.84 & & 31.71 & 22.10 & 37.23 & 29.30 & 28.75 & 21.40 \\
\hline & Service & 60.15 & 66.00 & 52.87 & & 68.24 & 76.70 & 58.25 & 60.70 & 65.76 & 59.10 \\
\hline \multirow[t]{3}{*}{2007} & Agriculture & 2.71 & 7.60 & 18.26 & & 0.05 & 1.10 & 4.41 & 9.00 & 5.56 & 18.30 \\
\hline & Industry & 37.01 & 25.50 & 29.03 & & 29.35 & 22.50 & 36.44 & 29.20 & 27.81 & 22.00 \\
\hline & Service & 60.28 & 66.90 & 52.71 & & 70.61 & 76.40 & 59.15 & 61.80 & 66.63 & 59.50 \\
\hline \multirow[t]{3}{*}{2008} & Agriculture & 2.51 & 7.20 & 17.78 & & 0.04 & 1.20 & 4.40 & 8.60 & 5.91 & 17.40 \\
\hline & Industry & 36.28 & 25.00 & 28.29 & & 27.33 & 22.50 & 36.12 & 28.90 & 27.90 & 22.60 \\
\hline & Service & 61.21 & 67.90 & 53.93 & & 72.63 & 76.20 & 59.48 & 62.40 & 66.18 & 59.70 \\
\hline \multirow[t]{3}{*}{2009} & Agriculture & 2.59 & 7.00 & 17.74 & 52.00 & 0.04 & 1.10 & 4.69 & 9.70 & 5.63 & 17.00 \\
\hline & Industry & 36.68 & 16.40 & 27.76 & 14.00 & 27.87 & 21.80 & 33.64 & 27.90 & 26.83 & 22.10 \\
\hline & Service & 60.73 & 76.60 & 54.50 & 34.00 & 72.09 & 77.10 & 61.67 & 62.30 & 67.54 & 60.70 \\
\hline \multirow[t]{3}{*}{2010} & Agriculture & 2.47 & 6.60 & 18.21 & 51.10 & 0.04 & & 3.87 & & 5.30 & \\
\hline & Industry & 38.27 & 17.00 & 27.16 & 22.40 & 27.63 & & 34.70 & & 28.07 & \\
\hline & Service & 59.26 & 76.40 & 54.64 & 26.60 & 72.33 & & 61.44 & & 66.63 & \\
\hline
\end{tabular}


From 1980 to 2010, the US service GDP share increased from $63.60 \%$ to $79 \%$ and its employment share increased from about $65.69 \%$ to $81.20 \%$.

China's relevant share of service GDP was $21.60 \%$ in 1980 to $43.24 \%$ in 2010 and employment share increased from $13.10 \%$ in 1980 to $34.60 \%$ in 2010 .

In Germany, service GDP was $56.50 \%$ in 1980 and $68.97 \%$ in 2010; and service employment increased from $51.90 \%$ in 1980 to $70.00 \%$ in 2010 .

In Japan, the service GDP was $57.86 \%$ in 1980 and $71.36 \%$ in 2010 ; the service employment was $54.00 \%$ in 1980 and $69.70 \%$ in 2010 .

In UK, it was $57.20 \%$ of service GDP in 1980 and $77.80 \%$ in 2010 ; and it was $58.90 \%$ of service employment in 1980 and $78.90 \%$ in 2010.

In South Korea, the service GDP was $50.72 \%$ in 1980 and $59.26 \%$ in 2010; the service employment was $37.00 \%$ in 1980 and $76.40 \%$ in 2010 .

In Singapore, the service GDP was $62.20 \%$ in 1980 and $72.09 \%$ in 2010; and the service employment was $62.59 \%$ in 1980 and $77.10 \%$ in 2009 .

In India, the service GDP was $40.32 \%$ in 1980 and $54.64 \%$ in 2010; the service employment was $24.00 \%$ in 2005 and $26.60 \%$ in 2010.

In Russia, the service GDP was $35.03 \%$ in 1990 and $61.67 \%$ in 2009; the service employment was $45.59 \%$ in 1990 and $62.30 \%$ in 2009 .

In Brazil, the service GDP was $45.16 \%$ in 1980 and $67.01 \%$ in 2011 ; the service employment was $49.29 \%$ in 1985 and $62.70 \%$ in 2011.

Table 4 b. Percentage changes of GDPs and jobs in service industry in different time periods

\begin{tabular}{|c|c|c|c|c|c|c|c|c|c|c|c|}
\hline & & China & US & Germany & Japan & UK & Korea & India & Singapore & Russia & Brazil \\
\hline \multicolumn{12}{|l|}{ 1980-1990 } \\
\hline \multirow[t]{3}{*}{ GDP $\% \triangle$} & Agriculture & -3.06 & -0.80 & -0.90 & -0.96 & -0.66 & -6.90 & -6.36 & -1.23 & 16.81 & -2.91 \\
\hline & Industry & -6.88 & -5.60 & -3.80 & -1.01 & -9.29 & 4.02 & 2.21 & -3.88 & 48.35 & -5.13 \\
\hline & Service & 9.94 & 6.50 & 4.70 & 1.96 & 9.94 & 2.87 & 4.16 & 5.12 & 35.03 & 8.05 \\
\hline \multirow[t]{3}{*}{ Job $\% \triangle$} & Agriculture & -8.60 & -0.69 & -1.70 & -3.20 & -0.50 & -16.11 & 0.00 & & 13.89 & 22.79 \\
\hline & Industry & 3.19 & -4.40 & -3.90 & -1.20 & -4.91 & 6.40 & 0.00 & & 40.00 & 22.70 \\
\hline & Service & 5.40 & 5.00 & 5.70 & 4.20 & 6.00 & 9.70 & 0.00 & & 45.59 & 54.50 \\
\hline \multicolumn{12}{|l|}{$1990-2000$} \\
\hline \multirow[t]{3}{*}{ GDP $\% \triangle$} & Agriculture & -12.05 & -0.91 & -0.39 & -0.53 & -0.54 & -3.82 & -6.00 & -0.23 & -10.38 & -2.50 \\
\hline & Industry & 4.58 & -4.46 & -6.79 & -7.05 & -4.61 & -0.09 & -0.49 & 2.49 & -10.40 & -10.96 \\
\hline & Service & 7.48 & 5.27 & 7.18 & 7.60 & 5.16 & 3.92 & 6.50 & -2.25 & 20.59 & 13.46 \\
\hline \multirow[t]{3}{*}{ Job $\% \triangle$} & Agriculture & -10.09 & -0.30 & -0.90 & -2.09 & -0.59 & -7.29 & 59.90 & & 0.61 & \\
\hline & Industry & 1.11 & -3.19 & -5.50 & -2.89 & -7.19 & -7.30 & 16.00 & & -11.60 & \\
\hline & Service & 9.00 & 3.61 & 6.20 & 4.90 & 8.20 & 14.50 & 24.00 & & 11.51 & \\
\hline \multicolumn{12}{|l|}{$2000-2010$} \\
\hline \multirow[t]{3}{*}{ GDP $\% \triangle$} & Agriculture & -4.97 & 0.00 & -0.31 & -0.41 & -0.22 & -1.92 & -4.82 & -0.06 & -2.57 & -0.30 \\
\hline & Industry & 0.75 & -3.65 & -0.28 & -3.54 & -5.28 & 0.18 & 1.16 & -7.20 & -3.25 & 0.34 \\
\hline & Service & 4.22 & 3.65 & 0.59 & 3.95 & 5.50 & 1.74 & 3.66 & 7.26 & 5.82 & -0.04 \\
\hline \multirow[t]{3}{*}{ Job $\% \triangle$} & Agriculture & -13.30 & -1.00 & -1.00 & -1.40 & -0.30 & -4.00 & -8.80 & & & \\
\hline & Industry & 6.20 & -6.50 & -5.10 & -5.90 & -6.00 & -11.10 & 6.40 & & & \\
\hline & Service & 7.10 & 6.90 & 6.20 & 6.60 & 5.80 & 15.20 & 2.60 & & & \\
\hline
\end{tabular}

Table 4b gives the percentage changes of GDP and Jobs between 1980-1990, 1990-2000 and 2000-2010 in three sectors in these selected countries. The data show how jobs and associated GDPs are shifted from other sectors to the service sector. 
The above analyses indicate that in around 30 years, in each of these selected counties, its service sector GDP and employment were increased significantly. In developing countries, the percentage increase of the GDP during the time period surpassed its percentage increase of the service employment. That means the whole economy's productivity was improved when the labor was shifted to the service sector (mostly from the agricultural sector). However, in developed countries, the percentage increase of the service GDP was slowed down clearly while the service employment increased significantly. That indicates that with the maturity of the economy, the shift of labor to the service sector (mostly from manufacturing) lowered a country's productivity.

As many other countries have experienced, such as China and India with their continued fast economic growth, more jobs will be shifted into the service sector. Currently, service employment is about $36 \%$ in China and $28 \%$ in India. In other well developed countries, this figure is between 70-80\%. Even in Brazil, it is above $60 \%$.

However, one needs to consider that both China and India are large populous countries and both are still under the industrialization stage; therefore their agriculture and industry sectors and its developments will remain to be essential to them. As a result, the shifts of labors to the service sector in these two countries in the coming decade will not be so dramatic as happened in western countries. The reasonable expectation will be about $45 \%$ of employment in the service sector in China and $38 \%$ in India by 2025 .

\subsection{China and Other Countries' Service Sector Distribution}

Table 5. Service distributions in different countries

\begin{tabular}{|c|c|c|c|c|c|c|c|c|c|}
\hline \multirow[b]{2}{*}{ Type of Service/Year } & \multicolumn{3}{|c|}{ China } & \multicolumn{3}{|c|}{ US } & \multicolumn{3}{|c|}{ Germany } \\
\hline & 2000 & 2005 & 2012 & 2000 & 2005 & 2012 & 2000 & 2005 & 2012 \\
\hline \multirow{3}{*}{$\begin{array}{l}\text { Distributive trade, repairs; } \\
\text { Transport; accommod., food serv. }\end{array}$} & $1,646,580$ & $2,882,806$ & $8,481,840$ & $1,651,480$ & $2,002,675$ & $2,385,914$ & 306,593 & 376,905 & 438,731 \\
\hline & & & & & & & & & \\
\hline & 0.43 & 0.38 & 0.37 & 0.23 & 0.21 & 0.20 & 0.24 & 0.23 & 0.21 \\
\hline Information and communication & & & & 545,176 & 729,989 & 920,463 & 79,995 & 91,951 & 121,236 \\
\hline \multirow[t]{2}{*}{ Financial and insurance activities } & 408,669 & 608,683 & $2,872,268$ & 722,739 & 952,102 & $1,074,935$ & 83,205 & 113,534 & 119,215 \\
\hline & 0.11 & 0.08 & 0.12 & 0.10 & 0.10 & 0.09 & 0.06 & 0.07 & 0.06 \\
\hline \multirow[t]{2}{*}{ Real estate activities } & 414,906 & 851,643 & $2,900,549$ & 983,658 & $1,339,263$ & $1,705,411$ & 208,075 & 265,102 & 365,260 \\
\hline & 0.11 & 0.11 & 0.13 & 0.14 & 0.14 & 0.14 & 0.16 & 0.16 & 0.18 \\
\hline $\begin{array}{l}\text { Prof., scientific, techn.; admin., } \\
\text { support serv. activities }\end{array}$ & & & & $1,074,308$ & $1,361,572$ & $1,768,531$ & 208,509 & 254,489 & 333,972 \\
\hline \multirow{3}{*}{$\begin{array}{l}\text { Public admin.; compulsory s.s.; } \\
\text { education; human health }\end{array}$} & & & & $1,939,589$ & $2,601,615$ & $3,460,375$ & 332,385 & 410,832 & 553,161 \\
\hline & & & & & & & & & \\
\hline & & & & 0.27 & 0.28 & 0.29 & 0.25 & 0.25 & 0.27 \\
\hline \multirow[t]{2}{*}{ Other service activities } & $1,401,241$ & $3,148,796$ & $8,885,992$ & 358,419 & 406,548 & 476,115 & 84,975 & 105,032 & 137,271 \\
\hline & 0.36 & 0.42 & 0.38 & 0.05 & 0.04 & 0.04 & 0.07 & 0.06 & 0.06 \\
\hline Total service output & $3,871,395$ & $7,491,927$ & $23,140,649$ & $7,275,369$ & $9,393,764$ & $11,791,744$ & $1,303,738$ & $1,617,845$ & $2,068,845$ \\
\hline
\end{tabular}

Table 5 lists the GDP in each service sector in these selected countries in 2000, 2005 and 2012. In China, percentage of GDP in "Distributive trade, repairs, transport, accommodation and food service" to its total service GDP was about 40\%; the GDP in "Financial and insurance activities" was about 10\%; it was about $10 \%$ in "Real estate activities" and 36\% in "Other services". China does not have relevant statistics in "Information and communication", "Professional scientific, technology, administrative, support service activities", and "Public administration, compulsory social service, education, human health". The lack of GDPs in these three sectors could be due to the differences in classifications. Most likely, these missed 
services should be included in "Other services". In the US, "Distributive trade, repairs, transport, accommodation and food service" composed about $20 \%$ of the total service GDP, and the share of "Public administration, compulsory social service, education, human health" was about $28 \%$. In South Korea, it was about $27 \%$ each in "Distributive trade, repairs, transport, accommodation and food service" and "Public administration, compulsory social service, education, human health". Compared with other countries, China's GDP shares in "Information and communication", "Professional scientific, technology, administrative, support service activities", and "Public administration, compulsory social service, education, human health" were too low.

Table 5. Continued

\begin{tabular}{|c|c|c|c|c|c|c|c|c|c|c|c|}
\hline \multirow[b]{2}{*}{ Type of Service/Year } & \multicolumn{3}{|c|}{ Japan } & \multicolumn{3}{|c|}{ United Kingdom } & \multicolumn{3}{|c|}{ South Korea } & \multicolumn{2}{|c|}{ Brazil } \\
\hline & 2000 & 2005 & 2012 & 2000 & 2005 & 2012 & 2000 & 2005 & 2012 & 2000 & 2005 \\
\hline \multirow{3}{*}{$\begin{array}{l}\text { Distributive trade, repairs; } \\
\text { Transport; accommod., food serv. }\end{array}$} & 596,584 & 765,666 & 872,843 & 183,097 & 229,458 & 267,462 & 124,637 & 152,479 & 197,519 & 139,866 & 285,671 \\
\hline & & & & & & & & & & & \\
\hline & 0.27 & 0.28 & 0.27 & 0.28 & 0.25 & 0.23 & 0.30 & 0.27 & 0.26 & 0.20 & 0.24 \\
\hline \multirow[t]{2}{*}{ Information and communication } & 163,637 & 202,767 & 250,010 & 61,084 & 78,772 & 92,214 & 32,215 & 45,956 & 51,879 & 44,580 & 84,399 \\
\hline & 0.07 & 0.07 & 0.08 & 0.09 & 0.09 & 0.08 & 0.08 & 0.08 & 0.07 & 0.06 & 0.07 \\
\hline \multirow[t]{2}{*}{ Financial and insurance activities } & 163,520 & 237,654 & 204,989 & 51,065 & 95,456 & 119,761 & 41,759 & 67,681 & 87,984 & 70,590 & 131,399 \\
\hline & 0.07 & 0.09 & 0.06 & 0.08 & 0.11 & 0.10 & 0.10 & 0.12 & 0.12 & 0.10 & 0.11 \\
\hline \multirow[t]{2}{*}{ Real estate activities } & 351,486 & 417,143 & 540,746 & 77,835 & 103,728 & 167,285 & 66,192 & 80,129 & 93,483 & 126,160 & 171,758 \\
\hline & 0.16 & 0.15 & 0.16 & 0.12 & 0.11 & 0.14 & 0.16 & 0.14 & 0.12 & 0.18 & 0.14 \\
\hline \multirow{2}{*}{$\begin{array}{l}\text { Prof., scientific, techn.; admin., } \\
\text { support serv. activities }\end{array}$} & & & & 98,549 & 132,720 & 175,777 & 31,516 & 48,031 & 71,572 & 58,213 & 99,979 \\
\hline & - & - & - & 0.15 & 0.15 & 0.15 & 0.08 & 0.08 & 0.09 & 0.08 & 0.08 \\
\hline \multirow{2}{*}{$\begin{array}{l}\text { Public admin.; compulsory s.s.; } \\
\text { education; human health }\end{array}$} & 354,661 & 424,118 & 519,444 & 152,871 & 221,559 & 281,877 & 97,644 & 156,240 & 227,996 & 160,787 & 291,761 \\
\hline & 0.16 & 0.16 & 0.16 & 0.23 & 0.24 & 0.24 & 0.24 & 0.27 & 0.30 & 0.23 & 0.24 \\
\hline \multirow[t]{2}{*}{ Other service activities } & 577,461 & 682,609 & 891,767 & 33,115 & 46,058 & 59,653 & 16,357 & 24,001 & 33,208 & 103,308 & 150,408 \\
\hline & 0.15 & 0.25 & 0.27 & 0.05 & 0.05 & 0.05 & 0.04 & 0.04 & 0.04 & 0.15 & 0.12 \\
\hline Total service output & $2,207,350$ & $2,729,956$ & $3,279,798$ & 657,616 & 907,751 & $1,164,029$ & 410,320 & 574,517 & 763,641 & 703,504 & $1,215,375$ \\
\hline
\end{tabular}

\subsection{China and Other Countries' Service Exports}

Table 6. China and Other Countries' Service Exports

\begin{tabular}{llllll}
\hline Country/Year & $\mathbf{2 0 0 5}$ & $\mathbf{2 0 0 6}$ & $\mathbf{2 0 0 7}$ & $\mathbf{2 0 0 8}$ & $\mathbf{2 0 0 9}$ \\
\hline China & $22,202,966,679$ & $27,731,770,927$ & $39,111,105,584$ & $47,031,906,970$ & $45,867,524,462$ \\
U.S & & $78,376,000,000$ & $94,572,000,000$ & $105,185,000,000$ & $107,753,000,000$ \\
Germany & $52,439,660,632$ & $59,743,463,711$ & $71,879,241,797$ & $84,268,513,759$ & $80,110,465,631$ \\
Japan & $20,238,044,590$ & $23,691,376,650$ & $26,364,949,708$ & $34,388,465,303$ & $36,030,158,696$ \\
U.K & $77,986,547,674$ & $87,793,954,826$ & $104,612,379,236$ & $103,817,519,347$ & $90,528,498,865$ \\
Korea & $9,922,400,000$ & $11,422,600,000$ & $15,308,000,000$ & $13,818,700,000$ & $12,860,800,000$ \\
Singapore & $10,292,072,657$ & $15,568,809,264$ & $17,587,466,451$ & $21,130,064,029$ & $20,397,593,593$ \\
India & $35,073,974,538$ & $47,423,914,835$ & $58,985,122,161$ & $73,484,592,821$ & $61,624,368,553$ \\
Russia & $6,833,560,000$ & $8,919,170,000$ & $12,366,658,000$ & $16,712,923,441$ & $14,005,980,410$ \\
Brazil & $6,435,168,000$ & $7,891,540,000$ & $11,483,100,000$ & $14,971,477,000$ & $14,404,566,000$ \\
\hline
\end{tabular}


Table 6 provides the total service exports of selected countries during 2005-2012. The US is ranked as \#1 in total exports in each of these years, UK \#2, and Germany \# 3. Although China is \#1 exporter in the world, its total service export was only ranked \#4, with the average annual service export of $\$ 151,655,129,608$, which was less than $30 \%$ that of the US (average annual service export of $\$ 519,507,750,000$ ).

Table 6. Continued

\begin{tabular}{lllll}
\hline Country/Year & $\mathbf{2 0 1 0}$ & $\mathbf{2 0 1 1}$ & $\mathbf{2 0 1 2}$ & Average \\
\hline China & $53,641,608,808$ & $60,690,749,652$ & $68,540,636,096$ & $45,602,283,647$ \\
U.S & $117,313,000,000$ & $131,949,000,000$ & $143,962,000,000$ & $111,301,428,571$ \\
Germany & $82,212,168,371$ & $90,128,275,179$ & $90,529,118,463$ & $76,413,863,443$ \\
Japan & $33,492,592,579$ & $38,588,091,031$ & $27,794,782,335$ & $30,073,557,612$ \\
U.K & $106,863,708,028$ & $115,778,917,690$ & $114,080,870,961$ & $100,182,799,578$ \\
Korea & $17,667,700,000$ & $19,471,000,000$ & $23,929,600,000$ & $15,550,100,000$ \\
Singapore & $23,018,927,888$ & $27,529,467,394$ & $28,532,110,873$ & $20,507,064,019$ \\
India & $75,037,123,007$ & $85,662,135,486$ & $95,891,807,671$ & $66,647,879,884$ \\
Russia & $14,965,290,000$ & $17,844,080,000$ & $19,901,820,000$ & $13,943,685,231$ \\
Brazil & $16,422,484,742$ & $20,198,895,520$ & $22,222,660,386$ & $14,253,736,456$ \\
\hline
\end{tabular}

Table 6a is the exports in Information, Communications and Technology during 2005-2012. China's annual ICT export was ranked as \# 5, less than that of India.

Table 6a. Information, communication and technology service exports (current US\$)

\begin{tabular}{llllll}
\hline Country/Year & $\mathbf{2 0 0 5}$ & $\mathbf{2 0 0 6}$ & $\mathbf{2 0 0 7}$ & $\mathbf{2 0 0 8}$ & $\mathbf{2 0 0 9}$ \\
\hline China & $22,202,966,679$ & $27,731,770,927$ & $39,111,105,584$ & $47,031,906,970$ & $45,867,524,462$ \\
U.S & & $78,376,000,000$ & $94,572,000,000$ & $105,185,000,000$ & $107,753,000,000$ \\
Germany & $52,439,660,632$ & $59,743,463,711$ & $71,879,241,797$ & $84,268,513,759$ & $80,110,465,631$ \\
Japan & $20,238,044,590$ & $23,691,376,650$ & $26,364,949,708$ & $34,388,465,303$ & $36,030,158,696$ \\
U.K & $77,986,547,674$ & $87,793,954,826$ & $104,612,379,236$ & $103,817,519,347$ & $90,528,498,865$ \\
Korea & $9,922,400,000$ & $11,422,600,000$ & $15,308,000,000$ & $13,818,700,000$ & $12,860,800,000$ \\
Singapore & $10,292,072,657$ & $15,568,809,264$ & $17,587,466,451$ & $21,130,064,029$ & $20,397,593,593$ \\
India & $35,073,974,538$ & $47,423,914,835$ & $58,985,122,161$ & $73,484,592,821$ & $61,624,368,553$ \\
Russia & $6,833,560,000$ & $8,919,170,000$ & $12,366,658,000$ & $16,712,923,441$ & $14,005,980,410$ \\
Brazil & $6,435,168,000$ & $7,891,540,000$ & $11,483,100,000$ & $14,971,477,000$ & $14,404,566,000$ \\
\hline
\end{tabular}

Table 6a. Continued

\begin{tabular}{lllll}
\hline Country/Year & $\mathbf{2 0 1 0}$ & $\mathbf{2 0 1 1}$ & $\mathbf{2 0 1 2}$ & Average \\
\hline China & $53,641,608,808$ & $60,690,749,652$ & $68,540,636,096$ & $45,602,283,647$ \\
U.S & $117,313,000,000$ & $131,949,000,000$ & $143,962,000,000$ & $111,301,428,571$ \\
Germany & $82,212,168,371$ & $90,128,275,179$ & $90,529,118,463$ & $76,413,863,443$ \\
Japan & $33,492,592,579$ & $38,588,091,031$ & $27,794,782,335$ & $30,073,557,612$ \\
U.K & $106,863,708,028$ & $115,778,917,690$ & $114,080,870,961$ & $100,182,799,578$ \\
Korea & $17,667,700,000$ & $19,471,000,000$ & $23,929,600,000$ & $15,550,100,000$ \\
Singapore & $23,018,927,888$ & $27,529,467,394$ & $28,532,110,873$ & $20,507,064,019$ \\
India & $75,037,123,007$ & $85,662,135,486$ & $95,891,807,671$ & $66,647,879,884$ \\
Russia & $14,965,290,000$ & $17,844,080,000$ & $19,901,820,000$ & $13,943,685,231$ \\
Brazil & $16,422,484,742$ & $20,198,895,520$ & $22,222,660,386$ & $14,253,736,456$ \\
\hline
\end{tabular}


Table $6 \mathrm{~b}$ is the percentage of Insurance and Finance export to the country's total service export during 2005-2012 for these selected countries. China has the lowest percentage with only $1.38 \%$ among all of these selected countries. UK is ranked as \#1 with $26.42 \%$, Singapore \#2 with $14.77 \%$, and US \# 3 with $14.38 \%$. Even other BRIC countries--Russia with $2.90 \%$, India with $5.47 \%$ and Brazil with $6.86 \%$-- were much higher than China's percentage.

Table 6b. Insurance and financial services exports (\% of service exports)

\begin{tabular}{llllllllll}
\hline Country/Year & $\mathbf{2 0 0 5}$ & $\mathbf{2 0 0 6}$ & $\mathbf{2 0 0 7}$ & $\mathbf{2 0 0 8}$ & $\mathbf{2 0 0 9}$ & $\mathbf{2 0 1 0}$ & $\mathbf{2 0 1 1}$ & $\mathbf{2 0 1 2}$ & Average \\
\hline China & 0.78 & 0.62 & 0.77 & 1.02 & 1.36 & 1.78 & 2.09 & 2.66 & 1.38 \\
U.S & 12.01 & 13.67 & 14.75 & 14.31 & 15.48 & 15.57 & 15.07 & 14.22 & 14.38 \\
Germany & 4.95 & 6.21 & 7.64 & 6.83 & 7.42 & 7.37 & 7.71 & 7.42 & 6.94 \\
Japan & 5.80 & 7.07 & 6.21 & 4.53 & 4.68 & 3.72 & 4.18 & 3.16 & 4.92 \\
U.K & 21.92 & 24.85 & 28.51 & 29.11 & 27.18 & 23.39 & 27.88 & 28.54 & 26.42 \\
Korea & 3.66 & 4.96 & 6.05 & 4.69 & 3.56 & 3.72 & 4.10 & 3.33 & 4.26 \\
Singapore & 12.56 & 13.62 & 15.99 & 14.20 & 15.94 & 15.55 & 15.32 & 14.97 & 14.77 \\
India & 3.99 & 5.00 & 5.64 & 5.52 & 5.52 & 6.50 & 6.38 & 5.23 & 5.47 \\
Russia & 2.47 & 2.70 & 3.54 & 3.12 & 3.07 & 3.02 & 2.48 & 2.80 & 2.90 \\
Brazil & 4.28 & 5.74 & 6.82 & 6.78 & 7.01 & 7.88 & 8.29 & 8.09 & 6.86 \\
\hline
\end{tabular}

Through the above analyses, it's clear that China needs to improve its service industry's competitiveness so that it can export more services. In particular, China needs to strengthen its services in sectors of Information and Communications and Insurance and Finance. More Chinese companies are doing business in other countries, and China's FDI outwards have been increasing over the years. There should be more demand for relevant services in overseas from Chinese companies, especially in the areas of insurance, finance and professional services such as legal and accounting.

\subsection{Correlation Analyses and Explanations}

The above table shows that all these variables are highly correlated. The correlation between Service GDP and Service Export is the highest, nearly 1, and also each service variable's correlation with the Total GDP is .99. These very high correlations of the service related variables with the world's economy (total GDP, total trade, and total export) indicate the importance of the service industry in the global economy.

Table 7. Correlations of the world's total GDP, trade, service GDP, export and service export

\begin{tabular}{lccccc}
\hline & Total GDP & Total Trade & Total Service GDP & Total Export & Total Service Export \\
\hline Total GDP & 1 & & & & \\
Total Trade & 0.97 & 1.00 & & & \\
Total Service GDP & 0.99 & 0.97 & 1.00 & 1.00 & 0.98 \\
Total Export & 0.97 & 1.00 & 1.00 & 0.98 & 1.00 \\
Total Service Export & 0.99 & 0.97 & & \\
\hline
\end{tabular}

Table 8a gives the correlations of GDPs of the selected countries between 1980-2013 and Table 8b is the ones between 2000-2013. Interestingly, China and US correlation was .85 during 1980-2013 and that raised to .90 in 2000-2013. On the other hand, the correlation between China and UK was reduced from .74 in 1980-2013 to .40 in 2000-2013. These changes show that the China and US economies have been increasingly correlated while the China and UK economies were the opposite. 
Table 8a. Correlations of GDPs of selected countries (1980-2013)

\begin{tabular}{lllllllllll}
\hline & China & US & Germany & Japan & UK & Korea & India & Singapore & Russia & Brazil \\
\hline China & 1 & & & & & & & & & \\
US & 0.85 & 1.00 & & & & & & & & \\
Germany & 0.82 & 0.95 & 1.00 & & & & & & & \\
Japan & 0.68 & 0.84 & 0.88 & 1.00 & & & & & & \\
UK & 0.74 & 0.96 & 0.94 & 0.76 & 1.00 & & & & & \\
Korea & 0.86 & 0.98 & 0.96 & 0.84 & 0.96 & 1.00 & & & & \\
India & 0.97 & 0.91 & 0.88 & 0.74 & 0.83 & 0.92 & 1.00 & & & \\
Singapore & 0.97 & 0.94 & 0.91 & 0.80 & 0.85 & 0.94 & 0.99 & 1.00 & & \\
Russia & 0.95 & 0.92 & 0.92 & 0.43 & 0.75 & 0.93 & 0.96 & 0.97 & 1.00 & \\
Brazil & 0.96 & 0.90 & 0.89 & 0.79 & 0.80 & 0.90 & 0.99 & 0.98 & 0.94 & 1.00 \\
\hline
\end{tabular}

Table 8 b. Correlations of GDPs of selected countries (2000-2013)

\begin{tabular}{|c|c|c|c|c|c|c|c|c|c|c|}
\hline & China & US & Germany & Japan & UK & Korea & India & Singapore & Russia & Brazil \\
\hline China & 1.00 & & & & & & & & & \\
\hline US & 0.90 & 1.00 & & & & & & & & \\
\hline Germany & 0.78 & 0.93 & 1.00 & & & & & & & \\
\hline Japan & 0.71 & 0.49 & 0.42 & 1.00 & & & & & & \\
\hline UK & 0.40 & 0.73 & 0.82 & -0.03 & 1.00 & & & & & \\
\hline South Korea & 0.83 & 0.95 & 0.86 & 0.40 & 0.78 & 1.00 & & & & \\
\hline India & 0.96 & 0.93 & 0.85 & 0.74 & 0.50 & 0.87 & 1.00 & & & \\
\hline Singapore & 0.99 & 0.92 & 0.81 & 0.72 & 0.47 & 0.87 & 0.98 & 1.00 & & \\
\hline Russia & 0.94 & 0.96 & 0.92 & 0.61 & 0.66 & 0.91 & 0.94 & 0.96 & 1.00 & \\
\hline Brazil & 0.95 & 0.88 & 0.83 & 0.79 & 0.44 & 0.82 & 0.99 & 0.97 & 0.94 & 1.00 \\
\hline
\end{tabular}

Table 9. Correlations of service exports of selected countries

\begin{tabular}{|c|c|c|c|c|c|c|c|c|c|c|}
\hline & China & US & Germany & Japan & UK & Korea & India & Singapore & Russia & Brazil \\
\hline China & 1.00 & & & & & & & & & \\
\hline US & 0.98 & 1.00 & & & & & & & & \\
\hline Germany & 0.96 & 0.94 & 1.00 & & & & & & & \\
\hline Japan & 0.93 & 0.88 & 0.96 & 1.00 & & & & & & \\
\hline UK & 0.86 & 0.81 & 0.83 & 0.86 & 1.00 & & & & & \\
\hline Korea & 0.98 & 0.98 & 0.93 & 0.91 & 0.83 & 1.00 & & & & \\
\hline Singapore & 0.98 & 0.99 & 0.93 & 0.88 & 0.78 & 0.97 & 1.00 & & & \\
\hline India & 0.98 & 1.00 & 0.92 & 0.88 & 0.79 & 0.97 & 1.00 & 1.00 & & \\
\hline Russia & 0.97 & 0.96 & 0.96 & 0.96 & 0.87 & 0.98 & 0.94 & 0.95 & 1.00 & \\
\hline Brazil & 0.98 & 1.00 & 0.94 & 0.90 & 0.79 & 0.98 & 0.99 & 0.99 & 0.96 & 1.00 \\
\hline
\end{tabular}

All these countries were highly corrected in service exports; particularly China had the extremely high correlations with virtually all other countries. One possible explanation is that a country's service export is affected by the trend of the whole world's demand for the service. This is true because the correlation between the world total service export and the total world GDP is .99 as Table 7 shows. Therefore, when the global economy is good and total demand for service export is high, all countries will benefit from that. 


\section{What China Can Learn from Other Countries and What It Should Do}

China is at the crucial stage of its economic development. It needs to identify new sources and engines to sustain its stable growth since previous approaches using mass exports and government investments have reached their limit. Stimulating domestic consumption and better developing its service industry have become China's new directions.

As Chen (2015) pointed out, currently manufacturing exports comprise $93 \%$ of China's total exports. Worldwide, that number is about $70 \%$ of total exports. Given China's development stage and employment needs, China still needs to continuously improve its manufacturing industry and export enough such products. In particular, China needs to become the strongest manufacturing country to sustain its economic growth. Interestingly, Prime Minister Li Keqiang used the same words to emphasize the importance and improvements of China's manufacturing industry in its annual report to China's Congress in March this year. He further outlined China's Manufacturing Industry 2025 similar to Germany's Manufacturing 4.0.

Besides stabilizing and improving its manufacturing industry, China certainly needs to expand and strengthen its service sector. As discussed in the previous sections, increasing importance and particular growing shares of employment and GDPs in services have been the worldwide trend that many developed countries have experienced. The issues germane to China are: how fast the service sector development can be and what will be potential problems, and how to solve them if service development is too fast.

Currently, China's service GDP is about $48.2 \%$ of its total GDP and employment is about $40 \%$ of total employment. In the past 12 years, China's annual GDP share in service rose by $0.47 \%$ and job share by $0.61 \%$. By 2025, China should expect about $56 \%$ GDP and $48 \%$ jobs in its service. By 2035, service GDP should be around $60 \%$ and employment around $55 \%$.

Table 10. Correlations of service exports of selected countries

\begin{tabular}{llllllll}
\hline & \multicolumn{2}{c}{$\mathbf{2 0 1 5}$} & \multicolumn{2}{c}{$\mathbf{2 0 2 5}$} & \multicolumn{2}{c}{$\mathbf{2 0 3 5}$} \\
& GDP $\%$ & Job \% & GDP \% & Job \% & GDP \% & Job \% \\
\hline Agriculture & 9 & 30 & 7 & 24 & 5 & 20 \\
Industry & 40.5 & 30 & 37 & 28 & 35 & 25 \\
Service & 50.5 & 40 & 56 & 48 & 60 & 55 \\
\hline
\end{tabular}

There will be some serious outcomes and problems if China grows its service sector too fast, i.e. its GDP and employment shares in services are too high, compared with the above proposed ranges.

1. Weightless. As some western countries have experienced, manufacturing outsourcing led to their economies to be weightless. The US government has called for manufacturers to come back to the US. If its GDP share in manufacturing becomes too low (less than 35\%, or even lower than 30\%), China' economy will face the weightless problem. Many better paid jobs in manufacturing will be lost and outsourced to other developing countries.

2. Cost Crises. As Baumol (1967) stated, shifting jobs from manufacturing to service may cause the cost crisis. The productivity in service is not rising but the average salary must be raised because of job market competition and the productivity's improvement in manufacturing, so the salary rises there. If such a crisis occurs, service firms will suffer and that will further negatively affect the whole economy.

3. Overall Productivity. When jobs shift from agriculture to manufacturing, the whole economy's productivity improves significantly. When too many jobs shift from manufacturing to service, the average productivity will be lower.

4. Low Salary. Usually, average salary in service is lower than in manufacturing. When many people move from manufacturing to service, their salary and income will be lower. That has been the case in many developed countries.

5. Competitiveness. The competitiveness is essential to an economy's strength. Globalization makes a country's competitiveness more important. Overdevelopment and shrinking of manufacturing sector will 
lead China to lose its competitiveness in the world. What are China's competitive advantages? More in manufacturing or in service?

6. Exports. Given China's large population and economic development stage, having enough exports will still be crucial to China's economy in coming decades, although contributions of the exports to China's GDP should be gradually lower. For more than two decades, China relied on increasing exports to support its rapid economic growths. In some years, the ratio of export to its GDP was above $40 \%$. In the future, China needs to rely more and more on its domestic consumption. But China still needs to maintain its fair share of the world's exports. If China cannot develop and implement an appropriate economic structure strategy and improve and strengthen both manufacturing and service sectors, it then will lose more world market share.

7. Mid-income trap. When a country uses its competitive advantages to advance its economy and reaches a certain income level, it may get stuck at that level because these previous competitive advantages have become obsolete and new ones could not be created. That is called the 'mid-income trap' as some newly industrialized countries like South Africa and Brazil have experienced. Their per capita GDP has remained around $\$ 10,000$ for more than a decade. These countries have suffered from low investment, slow growth in manufacturing, limited industrial diversification and poor labor market conditions. China's GDP per capita at present is around $\$ 7,000$ and will reach $\$ 10,000$ in about 6 years. To avoid such a mid-income trap will be a big challenge to China. Too rapid an expansion in service and shrinking in manufacturing will lead to such a trap, not solving the potential problem.

What should China do in order to maintain balanced, stable and sustainable economic development? As Chen (2015) emphasized, China must continuously strengthen and improve its manufacturing industry and make it the strongest sector. It needs to diversify its manufacturing industry and particularly develop sectors that consume less natural resources and emit less pollution. Also, it should develop more manufacturing products that can be used to directly substitute current mass imports and the ones representing future trends.

Expanding and developing the service industry will be an irreversible trend. Eventually, the GDP in service will surpass $60 \%$ of its total GDP and jobs in service will be above $50 \%$ of the total employment. But the issues facing China will be how soon that will be, what types of services will be needed, and what types of jobs will be necessary to sustain this growth.

As discussed in the previous sections, growing of the service sector and especially shrinking of manufacturing should be gradual, not dramatic. Social services and insurance/financial service should be given the highest priority. China now has more than 200 million seniors. Providing more and better services to these seniors should be the government and the society's responsibilities and that also creates large business opportunities. Insurance and financial service is quite underdeveloped in China and lags greatly when compared with other developed countries. Professional services, including legal, accounting, medical and psychological services, have huge potential for growth. Also, China needs to expand and improve its manufacturing services.

Another area is service export. As pointed out in the previous discussions, China's service export is far behind many other countries. Currently only about $7 \%$ of its total export is in service. Raising this ratio to $12-15 \%$ of its total export in the future should be reasonable and achievable.

\section{Conclusions}

This paper provides a comprehensive study and international comparisons of the service industry throughout the world. The data of the economic structure changes over the years for different countries, particularly the changes of the percentages of service GDPs and service employments in these countries show that the path of service industry development is based on each country's economic development level as well as its unique conditions. But generally speaking, the service industry becomes increasingly important when an economy advances. 
In order to avoid the potential mid-income trap, a country needs to continuously improve its manufacturing industry and particularly its competitiveness and diversity. It needs to develop and expand the service sectors which are not only highly needed but also productive and skill-intensive. Otherwise, the low average productivity and low average wage will slow the aggregate demand and the whole economy.

China is facing huge challenges and its economy is at a turning point. In order to maintain stable and sustainable economic growth, China needs to adjust its economic structure and better develop the service industry. It especially needs to expand and develop its professional, social and manufacturing services to better support its development.

China needs to adjust its economic development strategy and rely more on domestic consumption. It needs to focus more on innovations and institutional reforms to sustain economic growth. Sustaining and improving international trade and particularly exports will still be essential to China's economy and its future developments. Creating competitive advantages of its service industry and exporting more services will be crucial to China's future success.

\section{Limitations of the Study and Future Research}

This study focuses on comparing the service industry in a few countries, including China, US, Japan, Germany, United Kingdom, South Korean, Russia, Singapore, India, and Brazil. Further studies can cover more countries, including more developed and developing countries. In addition, one may select all developed countries together and compare their service industry or only compare the developing countries to see any significant differences in service industry and their comparative advantages. Each country is unique and different because of its history, culture, population, and economic conditions. But countries can learn from others and develop and improve its economic structure to better fit its conditions and raise its economic growth and people's welfare.

Also, one may focus on a specific service sector such as IT and Communications, Financial Service or Higher Education and conduct international comparisons of the sector. Such a specific service sector study can provide more detailed suggestions and help developing countries better learn from the others.

\section{References}

1. Baumol, William J (1967), "Macroeconomics of unbalanced growth: the anatomy of urban crisis," Am Econ Rev 57: pp. 415-426.

2. Baumol, William J., Ann Blackman, and Edward Wolf (1989), Productivity and American Leadership: The Long View, Cambridge, Massachusetts: MIT Press.

3. Bell, Daniel (1973), The Coming of Postindustrial Society: A Venture in Social Forecasting, New York: Basic Books.

4. Castells, Manuel and Yoku Aoyama (1993), "Paths towards the informational society: A comparative analysis of the transformation of employment structure in the G-7 countries, 1920-2005." BRIE Working Paper No. 61; Berkeley Roundtable on the International Economy; University of California, Berkeley.

5. Chen, Yueyun (2016), "International comparisons of agriculture industry - what China can learn from others?" International Journal of Agricultural Economics 1 (2): pp. 45-56.

6. Chen, Yueyun (2015), "China's path to sustainable, stable and rapid economic development: from the largest to the strongest manufacturing country," Journal of World Economic Research 4 (5-1): pp. 8-19.

7. Clark, Colin (1957), The Conditions of Economic Progress, London: Macmillan.

8. Cohen, Stephen, and John Zysman (1987), Manufacturing Matters: The Myth of the Postindustrial Economy. New York: Basic Books.

9. Conte A. and M. Vivarelli (2007), "Globalization and employment: imported skill biased technological change in developing countries," Jena Economic Research Papers 2007-009, Jena.

10.Denison, Edward (1985), Trends in American Economic Growth, 1929-1982. Washington. DC: Brookings Institution.. 
11.Dietrich, Andreas (2009), "Does growth cause structural change, or is it the other way around? a dynamic panel data analysis for seven OECD countries," Jena Economic Research Papers in Economics: pp. 2009-034.

12.Fisher, Allan GB (1939), "Production, primary, secondary and tertiary," Econ Rec 15: pp. 22-38.

13.Hansen, Gary D. and Edward C. Prescott (2002), "Malthus to show," American Economic Review 92 (4): pp. 12051217.

14.Krüger, Jens J (2008), "Productivity and structural change: a review of the literature," J Econ Surv 22: pp. 330-363.

15.Kuznets, Simon (1966), Modern Economic Growth, Rate Structure and Spread, New Haven and London: Yale University Press.

16.Huang, Yasheng (2010), "Debating China's economic growth: the Beijing consensus or the Washington consensus," Academy of Management Perspective: pp. 31-48.

17.Lewis, W. Arthur (1972), "Reflections on unlimited labor," in L. E. DiMarco (ed.) International Economics and Development (Essay in Honor of Raoul Prebisch), New York: Academic Press, pp. 75-96.

18.Li, Lanqing (2009), Breaking Through: The Birth of China's Opening-Up Policy, Oxford and New York: Oxford University Press.

19.Lin, Justin Yifu, Fan Cai, and Zhou Li (2003), The China Miracle: Development Strategy and Economic Reform, Hong Kong: Chinese University Press.

20.Lin, Justin Yifu (2012), The Quest for Prosperity: How Developing Countries Can Take Off, Princeton: Princeton University Press.

21.Lucas, Robert E (1988), "On the mechanics of economic development," J Monetary Econ 22: pp. 3-42.

22.McKinsey \& Company, Global Institute (2012), Manufacturing the Future: the Next Era of Global Growth and Innovation.

23.Monk, Peter (1989), Technological Change in the Information Economy, London: Pinter.

24.Nelson, Richard (1981), "Research of productivity growth and productivity differences: dead ends and new departures," Journal of Economic Literature 19 (9): pp. 1029-1064.

25.Oulton, Nicholas (2001), "Must the unbalanced growth rate decline? Baumol's unbalanced growth rate revisited," Oxford Economic Papers 53 (4): pp. 605-627.

26.Pelka, GW (2005), Wachstum und Strukturwandel, Metropolis-Verlag, Marburg.

27.Porat, M (1977), The Information Economy, Washington, DC, US Department of Commerce, Office of Telecommunications.

28.Potts, Jason and Tom Mandeville (2007), "Toward an evolutionary theory of innovation and growth in the service economy," Prometheus 25 (2): pp.147-159.

29.Rasiah, Rajah, Zhang Miao, and Kong Xin Xin (2013), "Can China's miraculous economic growth continue?" Journal of Contemporary Asia 43(2): pp. 295-313.

30.Romer, Paul M (1986), "Increasing returns and long-run growth," J Political Econ 94: pp. 1002-1037.

31.Romer, Paul M (1990), "Endogenous technological change," J Political Econ 98 (5): pp. 71-103.

32.Rostow, Walt Whitman (1960), "The Five stages of growth-a summary," The Stages of Economic Growth: A NonCommunist Manifesto. Cambridge: Cambridge University Press; pp. 4-16.

33.Sautter, Christian (1976), "Lefficacite et la rentabilite de leconomie francaise de 1954 a 1974," Economie et Statistique (Paris): pp. 68.

34.Sekhon, Harjit, et al. (2016), "A Cross-country study of service productivity," The Service Industries Journal 36 (5-6): pp. 223-238.

35.Silva, Eater G, and Aurora Teixeira (2008), "Surveying structural change: seminal contributions and a bibliometric account," Structure Chang Econ Dyn 19: pp. 273-300.

36.Smith, Adam (1776), "An inquiry into the nature and causes of the wealth of nations," in: Campbell RH, Skinner AS, Todd WB (eds) reprinted in 2 vols., Oxford: Clarendon Press.

37.Solow, Robert M (1956), "A Contribution to the theory of economic growth," Q J Econ 70: pp. 65-94.

38.Solow, Robert M (1957), "Technical change and the aggregate production function," Rev Econ Stat 39: pp. 312-320.

39.Stiglitz, Joseph and Linda J. Bilmes (2012), "The Book of jobs," Vanity Fair. 
40.Touraine, Alain (1969), La societe post-industrielle, Paris: Denoel.

41.Turgot, ARJ (1766), "Reflections on the formation and the distribution of wealth," in: Meek RL (eds): Turgot on Progress, sociology and economics; Cambridge: Cambridge University Press, pp. 119-134.

42.Wirtz, Jochen, Sven Tuzovic, and Michael Ehret (2015), "Global business services: increasing specialization and integration of the world economy as drivers of economic growth," Journal of Service Management 26 (4): pp. 565 587.

43.Zhu, Xiaodong (2012), "Understanding China's growth: past, present, and future," The Journal of Economic Perspectives 26 (4): pp.103-124. 